\title{
HARNACK-TYPE INEQUALITIES FOR EVOLUTION EQUATIONS
}

\author{
GILES AUCHMUTY AND DAVID BAO
}

(Communicated by Barbara Lee Keyfitz)

\begin{abstract}
Here we derive Harnack inequalities for nonnegative solutions of the porous medium equation and the $p$-diffusion equation. The method applies to functions obeying certain a priori evolution inequalities. The proofs are based on optimizing inequalities for the convective derivative of the function along a path.
\end{abstract}

\section{INTRODUCTION}

Harnack inequalities are well known for elliptic equations (see Harnack [8] and Gilbarg and Trudinger [6]). They are also known for parabolic equations since the work of Hadamard and Pini, published in 1954. In particular, for the heat equation

$$
\frac{\partial f}{\partial t}=\Delta f, \quad t>0,
$$

in Euclidean $\mathbb{R}^{n}$, positive solutions obey the following inequality

$$
f\left(x_{2}, t_{2}\right) \geq\left(\frac{t_{1}}{t_{2}}\right)^{n / 2} f\left(x_{1}, t_{1}\right) e^{-\left[\left|x_{2}-x_{1}\right|^{2} /\left(t_{2}-t_{1}\right)\right] / 4},
$$

where $t_{2}>t_{1}$, and $x_{i}=\left(x_{i}^{1}, \ldots, x_{i}^{n}\right)$. The derivation can be found in Moser [10], who studied the more general equation $\frac{\partial f}{\partial t}=[\Delta-q(x)] f$. An extension of (1.2) to complete manifolds with nonnegative Ricci curvature was given by $\mathrm{Li}$ and $\mathrm{Yau}[9]$.

More recently there has been extensive work on some other types of Harnack inequalities and Hölder estimates for nonlinear parabolic equations. For an overview, see the survey lectures by DiBenedetto [3], and also DiBenedetto and Friedman [4].

In this paper, we shall describe some different pointwise bounds on nonnegative solutions of nonlinear parabolic equations which obey certain a priori estimates. Our approach is short and geometric and is based on an ingenious

Received by the editors November 30, 1992.

1991 Mathematics Subject Classification. Primary 35K55.

The first author's research was partially supported by NSF Grant DMS 8901477. 
variational argument due originally to $\mathrm{Li}$ and Yau [9] and in a form described by Richard Hamilton [7].

\section{THE POROUS MEDIUM EQUATION AND THE HEAT EQUATION}

In this section the equations for porous media and heat conduction will be used to illustrate the method for obtaining pointwise bounds like (1.2). We shall use various known a priori bounds for the equations, and we shall optimize along paths in a manner similar to that done by Hamilton [7]. For the rest of the paper, let $|V|$ and $U \cdot V$ denote, respectively, the Euclidean norm and the usual inner product on $\mathbb{R}^{n}$.

The porous medium equation (for a survey, see Aronson [1]) is

$$
\frac{\partial u}{\partial t}=\Delta\left(u^{M}\right), \quad t>0, x \in \mathbb{R}^{n} .
$$

Throughout this paper, we shall confine our attention to

$$
M>M_{0}(n):=\max \left\{0,1-\frac{2}{n}\right\} .
$$

Under the change of dependent variable

$$
f:=\left(\frac{M}{M-1}\right) u^{M-1}, \quad M \neq 1,
$$

the equation becomes

$$
\frac{\partial f}{\partial t}=(M-1) f \Delta f+|\nabla f|^{2} .
$$

The case $M=2$ was discussed by Hamilton [7] as the migration equation. For nonnegative weak solutions of (2.3), Aronson and Bénilan [2] applied a maximum principle to obtain

$$
\triangle f \geq \frac{-k}{t}, \quad k:=\frac{1}{\left(M-1+\frac{2}{n}\right)} .
$$

First specialize to the case $M>1$. Multiplying $(2.4)$ by $(M-1) f$ and using (2.3), it follows that

$$
\frac{\partial f}{\partial t}+[(M-1) k] \frac{f}{t} \geq|\nabla f|^{2} .
$$

Adding $2(\nabla f) \cdot W+|W|^{2}$ to both sides of $(2.5)$, where $W$ is an arbitrary vector field on $\mathbb{R}^{n}$, leads to

$$
\frac{\partial f}{\partial t}+[(M-1) k] \frac{f}{t}+2(\nabla f) \cdot W+|W|^{2} \geq 0 .
$$

Now choose $W$ judiciously. Let $x(t)$ be any $C^{1}$ path in $\mathbb{R}^{n}$ such that $x\left(t_{1}\right)=x_{1}$ and $x\left(t_{2}\right)=x_{2}$; let $\dot{x}$ denote its velocity field. In (2.6), set $W=\frac{1}{2} \dot{x}$ and use the chain rule $\frac{d f}{d t}=\frac{\partial f}{\partial t}+(\nabla f) \cdot \dot{x}$; then

$$
\frac{d f}{d t}+[(M-1) k] \frac{f}{t}+\frac{1}{4}|\dot{x}|^{2} \geq 0 .
$$


Let $\mu:=(M-1) k$; then use the integrating factor $t^{\mu}$ in (2.7) to obtain

$$
t_{1}^{\mu} f\left(x_{1}, t_{1}\right) \leq t_{2}^{\mu} f\left(x_{2}, t_{2}\right)+\frac{1}{4} \int_{t_{1}}^{t_{2}} t^{\mu}|\dot{x}|^{2} d t
$$

for all $C^{1}$ paths $x(t)$ such that $x\left(t_{1}\right)=x_{1}$ and $x\left(t_{2}\right)=x_{2}$.

The inequality (2.8) can be sharpened by minimizing the integral on the right, as a functional on the allowable class of paths $x(t)$. The convexity of $|\dot{x}|^{2}$ implies that the minimum of this functional is attained by the solution of the relevant Euler-Lagrange equation. Using straightforward classical analysis, this minimizer is given by the parametrized curve

$$
x(t)=\left(\frac{x_{2}-x_{1}}{t_{2}^{\delta}-t_{1}^{\delta}}\right) t^{\delta}+\left(\frac{x_{1} t_{2}^{\delta}-x_{2} t_{1}^{\delta}}{t_{2}^{\delta}-t_{1}^{\delta}}\right), \quad \delta:=1-\mu .
$$

When $t_{2}>t_{1}>0$, the minimum value of the integral is $\frac{\delta}{\left(t_{2}^{\delta}-t_{1}^{\delta}\right)}\left|x_{2}-x_{1}\right|^{2}$. The resulting Harnack inequality is

$$
t_{1}^{\mu} f\left(x_{1}, t_{1}\right) \leq t_{2}^{\mu} f\left(x_{2}, t_{2}\right)+\frac{1}{4} \frac{\delta}{\left(t_{2}^{\delta}-t_{1}^{\delta}\right)}\left|x_{2}-x_{1}\right|^{2},
$$

where

$$
\begin{aligned}
& t_{2}>t_{1}>0, \quad k:=\frac{1}{\left(M-1+\frac{2}{n}\right)}, \\
& 0<\mu:=(M-1) k<1, \quad \delta:=1-\mu=\frac{2 k}{n} .
\end{aligned}
$$

Equation (2.10a) may be rearranged to yield

$$
f\left(x_{2}, t_{2}\right) \geq\left(\frac{t_{1}}{t_{2}}\right)^{\mu}\left[f\left(x_{1}, t_{1}\right)-\frac{\delta}{4} \frac{\left|x_{2}-x_{1}\right|^{2}}{t_{2}^{\delta}-t_{1}^{\delta}} \frac{1}{t_{1}^{\mu}}\right],
$$

which provides lower bounds on the decay of nonnegative solutions of (2.3) [for $M>1$ ] in a cusped region above $\left(x_{1}, t_{1}\right)$. When $f$ is positive, it may be restated as

$$
f\left(x_{2}, t_{2}\right) \geq\left(\frac{t_{1}}{t_{2}}\right)^{\mu} f\left(x_{1}, t_{1}\right)\left[1-\frac{\delta}{4} \frac{\left|x_{2}-x_{1}\right|^{2}}{t_{2}^{\delta}-t_{1}^{\delta}} \frac{1}{f\left(x_{1}, t_{1}\right)} \frac{1}{t_{1}^{\mu}}\right]
$$

in order to facilitate comparison with $\S 3$. We note that these lower bounds on $f$ yield, upon substituting (2.2) with $M>1$, lower bounds on the solutions $u$ of the original porous medium equation (2.1).

The heat equation (1.1) was excluded from the above analysis by the requirement $M \neq 1$. For (1.1) [that is, (2.1) with $M=1$ ], Aronson and Bénilan [2] showed that $\Delta f \geq \frac{-n}{2 t} f$ for nonnegative solutions $f$; consequently, the analogue of $(2.5)$ is

$$
\frac{\partial f}{\partial t}+\frac{n}{2 t} f \geq 0 \text {. }
$$

Due to the absence of $|\nabla f|^{2}$ on the right-hand side, we cannot 'complete the square' as before. Happily, using the methods of [2] (or see Li and Yau [9]), (2.12) can be sharpened to

$$
\frac{\partial f}{\partial t}+\frac{n}{2 t} f \geq \frac{|\nabla f|^{2}}{f}
$$


for positive solutions of the heat equation on any complete Riemannian manifold with nonnegative Ricci curvature. In Euclidean space, (2.13) can be verified directly by using the fundamental solution. Now add $2(\nabla f) \cdot W+f|W|^{2}$ to both sides of (2.13) and observe that the right-hand side becomes $f\left|\frac{\nabla f}{f}+W\right|^{2}$, which is nonnegative for any vector field $W$. Proceeding as for the porous medium equation and choosing $W=\frac{1}{2} \dot{x}$, we find that the analogue of $(2.7)$ is

$$
\frac{1}{f} \frac{d f}{d t}+\frac{n}{2 t}+\frac{1}{4}|\dot{x}|^{2} \geq 0 \text {. }
$$

Integration of this yields

$$
\log \left[t_{1}^{\mu} f\left(x_{1}, t_{1}\right)\right] \leq \log \left[t_{2}^{\mu} f\left(x_{2}, t_{2}\right)\right]+\frac{1}{4} \int_{t_{1}}^{t_{2}}|\dot{x}|^{2} d t, \quad \mu:=\frac{n}{2},
$$

for all $C^{1}$ paths $x(t)$ such that $x\left(t_{1}\right)=x_{1}$ and $x\left(t_{2}\right)=x_{2}$. This time the integral on the right-hand side has the minimum value $\frac{\left|x_{2}-x_{1}\right|^{2}}{t_{2}-t_{1}}$. Substituting this into (2.15) and exponentiating, we obtain the previous lower bound (1.2).

So far, we have studied (2.1) for the cases $M>1$ and $M=1$. The case $M_{0}(n)<M<1$ will be treated in $\S 6$.

\section{General Harnack-type inequalities}

The derivation of the inequalities (2.9) and (1.2) depended essentially on a priori bounds of the form

$$
\frac{\partial f}{\partial t}+a(t) f \geq \frac{C|\nabla f|^{p}}{f^{r}},
$$

where $C$ is a positive constant and $a$ is a locally integrable function. The following result shows how pointwise lower bounds can be obtained for positive (if $r \neq 0$ ) or nonnegative (if $r=0$ ) functions which obey (3.1).

Theorem 1. Let $\Omega$ be an open convex set in $\mathbb{R}^{n}$ and $f: \Omega \times(0, T) \rightarrow[0, \infty)$ be a $C^{1}$ function which obeys (3.1) with $C>0, p>1$, and $a$ in $L_{\mathrm{loc}}^{1}[0, T]$. For all $x_{1}, x_{2}$ in $\Omega$ and $0<t_{1}<t_{2}<T$, we have the following inequalities:

(i) when $r=p-1$, then

$$
f\left(x_{2}, t_{2}\right) \geq \frac{e^{A\left(t_{1}\right)}}{e^{A\left(t_{2}\right)}} f\left(x_{1}, t_{1}\right) e^{-\xi\left|x_{2}-x_{1}\right|^{q} /\left(t_{2}-t_{1}\right)^{q-1}}
$$

holds for positive $f$;

(ii) when $r>p-1$, then

$$
f\left(x_{2}, t_{2}\right) \geq \frac{e^{A\left(t_{1}\right)}}{e^{A\left(t_{2}\right)}} f\left(x_{1}, t_{1}\right)\left(1+m \xi\left|x_{2}-x_{1}\right|^{q} I^{1-q}\left[f\left(x_{1}, t_{1}\right)\right]^{m} e^{m A\left(t_{1}\right)}\right)^{\frac{-1}{m}}
$$

holds for positive $f$;

(iii) when $r<p-1$, then

$$
\left[f\left(x_{2}, t_{2}\right)\right]^{|m|} \geq\left(\frac{e^{A\left(t_{1}\right)}}{e^{A\left(t_{2}\right)}}\right)^{|m|}\left(\left[f\left(x_{1}, t_{1}\right)\right]^{|m|}-\frac{|m| \xi\left|x_{2}-x_{1}\right|^{q} I^{1-q}}{e^{|m| A\left(t_{1}\right)}}\right)
$$

holds for nonnegative $f$ and, in case $f$ is positive and the quantity inside the large parentheses is nonnegative, we have

$$
f\left(x_{2}, t_{2}\right) \geq \frac{e^{A\left(t_{1}\right)}}{e^{A\left(t_{2}\right)}} f\left(x_{1}, t_{1}\right)\left(1-\frac{|m| \xi\left|x_{2}-x_{1}\right|^{q} I^{1-q}}{\left[f\left(x_{1}, t_{1}\right)\right]^{|m|} e^{|m| A\left(t_{1}\right)}}\right)^{\frac{1}{|m|}} .
$$


Here, $q:=\frac{p}{p-1}$ is the conjugate index to $p, m:=\frac{r}{p-1}-1, \xi:=\frac{1}{q}\left(\frac{1}{p C}\right)^{q-1}$, $A(t)$ is any antiderivative of $a(t)$, and $I:=\int_{t_{1}}^{t_{2}} e^{m(p-1) A(t)} d t$.

Proof. To obtain these results we use Young's inequality in the form

$$
\frac{1}{p}|Y|^{p}+\frac{1}{q}|Z|^{q} \geq Y \cdot Z,
$$

where $1<p<\infty$ is any real number, $q=\frac{p}{p-1}$ is its conjugate index (that is, $\frac{1}{p}+\frac{1}{q}=1$ ), and $Y, Z$ are arbitrary vectors in $\mathbb{R}^{n}$. We pause to explain why Young's inequality is to be used here instead of a generalization of the completing-the-square procedure in $\S 2$. Recall that we completed the square there by adding $2(\nabla f) \cdot W+|W|^{2}$ to both sides of $(2.5)$. However, we could have added $-(\nabla f) \cdot W+\frac{1}{2}\left|\frac{1}{\sqrt{2}} W\right|^{2}$ instead; and the right-hand side of the resulting inequality would be $2\left[\frac{1}{2}|\nabla f|^{2}-(\nabla f) \cdot\left(\frac{W}{2}\right)+\frac{1}{2}\left|\frac{W}{2}\right|^{2}\right]$, which is nonnegative by the above Young's inequality. Now proceed as before, but this time specify $W$ as $-\dot{x}$ rather than $\frac{1}{2} \dot{x}$; we would again obtain (2.7). For (3.1), completing the $p$ th power optimally yields the analogue of (2.7); but we choose to use Young's inequality because, in retrospect, it achieves the same result much more elegantly.

Let $\phi$ (resp. $W$ ) be any scalar- (resp. vector-) valued continuous function defined on $\Omega \times(0, T)$ and add $-(\nabla f) \cdot W+\frac{1}{q}|\phi W|^{q}$ to both sides of (3.1). The right-hand side of the resulting inequality, upon the choice $\phi=\left(\frac{f^{r}}{p C}\right)^{\frac{1}{p}}$, simplifies to the quantity $\left(\frac{p C}{f^{r}}\right)\left[\frac{1}{p}|Y|^{p}-Y \cdot Z+\frac{1}{q}|Z|^{q}\right]$ with $Y=\nabla f$ and $Z=$ $\left(\frac{f^{r}}{p C}\right) W$. By (3.5), this is pointwise nonnegative on $\Omega \times(0, T)$; so we have

$$
\frac{\partial f}{\partial t}+a(t) f-(\nabla f) \cdot W+\xi f^{r /(p-1)}|W|^{q} \geq 0, \quad \xi:=\frac{1}{q}\left(\frac{1}{p C}\right)^{q-1}
$$

for any vector field $W$ defined on $\Omega \times(0, T)$.

Given $x_{1}, x_{2}$ in $\Omega$, let $\left\{x(t): t_{1} \leq t \leq t_{2}\right\}$ be a $C^{1}$ curve in $\Omega$ with $x\left(t_{1}\right)=x_{1}$ and $x\left(t_{2}\right)=x_{2}$. Choose $W=-\dot{x}$, and apply the chain rule to the convective derivative $\frac{d}{d t} f(x(t), t)$ of $f$ along the curve; then (3.6) becomes

$$
\begin{aligned}
& \frac{d}{d t} f(x(t), t)+a(t) f(x(t), t) \\
& \quad \geq-\xi[f(x(t), t)]^{m+1}|\dot{x}(t)|^{q}, \quad m+1:=\frac{r}{p-1} .
\end{aligned}
$$

We shall obtain different estimates corresponding to the three cases: $m=0$, $m>0, m<0$. In what follows, let

$$
A(t):=\int^{t} a(s) d s
$$

be any antiderivative of $a$.

(i) $m=0$, that is, $r=p-1$. For positive $f$, equation (3.7) becomes

$$
\frac{1}{f} \frac{d f}{d t}+a(t) \geq-\xi|\dot{x}|^{q}
$$

and we see that the situation here is analogous to the heat equation case (2.12). Upon integration, (3.8) yields

$$
\log \left[f\left(x_{2}, t_{2}\right)\right]+A\left(t_{2}\right)+\xi \int_{t_{1}}^{t_{2}}|\dot{x}|^{q} d t \geq \log \left[f\left(x_{1}, t_{1}\right)\right]+A\left(t_{1}\right) .
$$


This holds for any $C^{1}$ path as above. The integral here is minimized by a straight line and has the value $\frac{\left|x_{2}-x_{1}\right|^{q}}{\left(t_{2}-t_{1}\right)^{q-1}}$ whenever $t_{2}>t_{1}>0$. Substitute this into (3.9), exponentiate, and rearrange; then we get

$$
f\left(x_{2}, t_{2}\right) \geq \frac{e^{A\left(t_{1}\right)}}{e^{A\left(t_{2}\right)}} f\left(x_{1}, t_{1}\right) e^{-\xi\left|x_{2}-x_{1}\right|^{q} /\left(t_{2}-t_{1}\right)^{q-1}},
$$

which is $(3.2)$.

(ii) $m>0$, that is, $r>p-1$. Multiplying (3.7) by the integrating factor $e^{A(t)}$ gives $\frac{d}{d t}\left[f e^{A}\right] \geq-\xi f^{m+1} e^{A}|\dot{x}|^{q}$. For positive $f$, this can be written as

$$
\frac{-m}{\left(f e^{A}\right)^{m+1}} \frac{d}{d t}\left(f e^{A}\right) \leq m \xi e^{-m A}|\dot{x}|^{q} .
$$

Upon integration, this becomes

$$
\left[f\left(x_{2}, t_{2}\right) e^{A\left(i_{2}\right)}\right]^{-m} \leq\left[f\left(x_{1}, t_{1}\right) e^{A\left(t_{1}\right)}\right]^{-m}+m \xi \int_{t_{1}}^{t_{2}} e^{-m A}|\dot{x}|^{q} d t .
$$

By a straightforward exercise in the classical calculus of variations, the minimum of the integral on the right-hand side is found to occur at the path

$$
x(t)=x_{1}+\frac{x_{2}-x_{1}}{I} \int_{t_{1}}^{t} e^{m(p-1) A(s)} d s, \quad I:=\int_{t_{1}}^{t_{2}} e^{m(p-1) A(t)} d t .
$$

That integral then has the minimal value

$$
\left|x_{2}-x_{1}\right|^{q} I^{1-q} \text {. }
$$

Substituting this into (3.11) and after some rearrangement, we obtain the inequality

$$
f\left(x_{2}, t_{2}\right) \geq \frac{e^{A\left(t_{1}\right)}}{e^{A\left(t_{2}\right)}} f\left(x_{1}, t_{1}\right)\left(1+m \xi\left|x_{2}-x_{1}\right|^{q} I^{1-q}\left[f\left(x_{1}, t_{1}\right)\right]^{m} e^{m A\left(t_{1}\right)}\right)^{\frac{-1}{m}},
$$

which is $(3.3)$.

(iii) $m<0$, that is, $r<p-1$. As in (ii), we multiply (3.7) by the integrating factor $e^{A(t)}$ and rearrange. The analogue of (3.10) is now

$$
\frac{-m}{\left(f e^{A}\right)^{m+1}} \frac{d}{d t}\left(f e^{A}\right) \geq m \xi e^{-m A}|\dot{x}|^{q} .
$$

This holds for positive $f$ when $m \neq-1$ (that is, $r \neq 0$ ), and for nonnegative $f$ when $m=-1(r=0)$. Upon integration, (3.13) gives

$$
\left[f\left(x_{1}, t_{1}\right) e^{A\left(t_{1}\right)}\right]^{|m|} \leq\left[f\left(x_{2}, t_{2}\right) e^{A\left(t_{2}\right)}\right]^{|m|}+|m| \xi \int_{t_{1}}^{t_{2}} e^{-m A}|\dot{x}|^{q} d t .
$$

Using the minimizing path given in (3.12) and rearranging, we obtain

$$
\left[f\left(x_{2}, t_{2}\right)\right]^{|m|} \geq\left(\frac{e^{A\left(t_{1}\right)}}{e^{A\left(t_{2}\right)}}\right)^{|m|}\left(\left[f\left(x_{1}, t_{1}\right)\right]^{|m|}-\frac{|m| \xi\left|x_{2}-x_{1}\right|^{q} I^{1-q}}{e^{|m| A\left(t_{1}\right)}}\right),
$$

which is $(3.4 \mathrm{a})$. For positive $f$, this can be rewritten as

$$
\left[f\left(x_{2}, t_{2}\right)\right]^{|m|} \geq\left(\frac{e^{A\left(t_{1}\right)}}{e^{A\left(t_{2}\right)}}\right)^{|m|}\left[f\left(x_{1}, t_{1}\right)\right]^{|m|}\left(1-\frac{|m| \xi\left|x_{2}-x_{1}\right|^{q} I^{1-q}}{\left[f\left(x_{1}, t_{1}\right)\right]^{|m|} e^{|m| A\left(t_{1}\right)}}\right) .
$$


When the quantity inside the large parentheses is nonnegative, taking the $|m|$ th root of both sides yields $(3.4 b)$.

Inequalities (3.2) and (3.3) show that if $f\left(x_{1}, t_{1}\right)>0$ and $f$ obeys (3.1) with $r \geq p-1>0$, then $f\left(x_{2}, t_{2}\right)>0$ for all $x_{2} \in \Omega$ and $t_{2}>t_{1}$; that is, we have infinite speed of propagation of information. When $r<p-1,(3.4)$ does not provide such a result; this is expected because porous medium flows (with $M>1$ ) fall under case (iii) of the theorem (see the remarks at the end of this section), and it is known [1] that disturbances in such flows propagate with a finite speed.

For this theorem, $f$ was assumed to be $C^{1}$ and (3.1) to hold pointwise. For a number of important applications, the functions will not be $C^{1}$ and the evolution inequality (3.1) only holds in a distributional sense. The only place where the regularity assumptions on $f$ are necessary is in justifying the chain rule

$$
\frac{d}{d t} f(x(t), t)=\frac{\partial f}{\partial t}+(\nabla f) \cdot \dot{x}
$$

in going from (3.6) to (3.7).

To extend this theorem to weak solutions of (3.1), we shall use some definitions and results from Marcus and Mizel [11] to justify (3.15). Let us assume that: $\Omega$ is an open convex set in $\mathbb{R}^{n}$;

(F1) $f: \Omega \times(0, T) \rightarrow[0, \infty)$ is a continuous function and $f \in$ $W_{\text {loc }}^{1,1}(\Omega \times(0, T))$;

(F2) $0<t_{1}<t_{2}<T$ and, the set of points in $\Omega \times\left[t_{1}, t_{2}\right]$ where $f$ does not have a classical total differential, when orthogonally projected onto each coordinate axis, has 1-dimensional Lebesgue measure equal to 0 ;

(F3) $f$ is absolutely continuous on every $C^{1}$ curve $x(t), t_{1} \leq t \leq t_{2}$, lying in $\Omega$; and

(F4) $f$ obeys (3.1) with $C>0$ and the function $a$ is in $L^{1}\left(\left[t_{1}, t_{2}\right]\right)$.

Theorem 2. Suppose $\Omega$ is an open convex set in $\mathbb{R}^{n}, f$ obeys (F1)-(F4), and (3.1) holds in a distributional sense with $p>1$. Then for all $x_{1}, x_{2}$ in $\Omega$ and $0<t_{1}<t_{2}<T$, (i)-(iii) of Theorem 1 hold.

Proof. In the proof of Theorem 1, we must now replace classical derivatives of $f$ by distributional derivatives. The fact that the chain rule (3.15) holds follows from Theorem 4.1 in [11] because the quantity $(\nabla f) \cdot \dot{x}$ will be $L^{1}$ on each $C^{1}$ curve $x(t)$. The validity of $\int_{t_{1}}^{t_{2}} \frac{d}{d t} h(x(t), t) d t=h\left(x_{2}, t_{2}\right)-h\left(x_{1}, t_{1}\right)$ follows from Lebesgue's theorem and (F3). The variational arguments remain unchanged.

Marcus and Mizel [11] have shown, in particular, that any locally Lipshitz function $f$ on $\Omega \times(0, T)$ will obey (F2) and (F3) of the above list of assumptions.

A further generalization of our result can be obtained for functions $f$ obeying evolution inequalities of the form

$$
\frac{\partial f}{\partial t}+a(t) f \geq \phi(\nabla f), \quad \text { on } \Omega \times(0, T)
$$


where $\phi: \mathbb{R}^{n} \rightarrow \mathbb{R}$ is a convex function. In this case (3.7) becomes

$$
\frac{d}{d t} f(x(t), t)+a(t) f(x(t), t) \geq-\phi^{*}(-\dot{x}(t)),
$$

where $\phi^{*}$ is the conjugate convex function of $\phi$. Multiplying (3.17) by $e^{A}$ and integrating, we find that

$$
f\left(x_{2}, t_{2}\right) e^{A\left(t_{2}\right)}-f\left(x_{1}, t_{1}\right) e^{A\left(t_{1}\right)} \geq-\int_{t_{1}}^{t_{2}} e^{A(t)} \phi^{*}(-\dot{x}(t)) d t .
$$

Whenever we can evaluate the minimum value of this integral, lower bounds on nonnegative solutions of (3.16) can be found.

Finally, we remark that for the heat equation, (3.1) holds with $C=1, p=2$, and $r=1$, so case (i) of our theorem applies and the resulting estimate (3.2) becomes the Harnack inequality (1.2) when we set $a(t)=\frac{n}{2 t}$. For the porous medium equation with $M>1$, (3.1) holds with $C=1, p=2$, and $r=0$. Case (iii) of our theorem applies, and the resulting inequalities (3.4a) and (3.4b) become (2.11a) and (2.11b), respectively. To see this, observe that $m=-1$ and $a(t)=\frac{(M-1) k}{t}$, where $k$ is defined by $(2.4)$.

\section{THE $p$-DIFFUSION EQUATION}

These methods will now be applied to derive Harnack-type inequalities for the $p$-diffusion equation

$$
\frac{\partial u}{\partial t}=\operatorname{div}\left(|\nabla u|^{p-2} \nabla u\right), \quad(x, t) \in \mathbb{R}^{n} \times(0, \infty) .
$$

When $p \neq 2$, make the change of dependent variable

$$
f:=\frac{1}{\gamma} u^{\gamma}, \quad \gamma:=\frac{p-2}{p-1}
$$

then (4.1) becomes

$$
\frac{\partial f}{\partial t}=\gamma f \operatorname{div}\left(|\nabla f|^{p-2} \nabla f\right)+|\nabla f|^{p} .
$$

Esteban and Vazquez [5] have shown that for nonnegative solutions of (4.3), there exists a positive constant $K(p, n)$ depending only on $p$ and $n$ such that

$$
\operatorname{div}\left(|\nabla f|^{p-2} \nabla f\right) \geq \frac{-K}{t},
$$

provided that

$$
p>2\left(\frac{n}{n+1}\right) .
$$

Multiplying (4.4a) by $\gamma f$ and using (4.3) gives

$$
\frac{\partial f}{\partial t}+\left(\frac{\gamma K}{t}\right) f \geq|\nabla f|^{p}
$$

This evolution inequality has the form (3.1) with $C=1, r=0$, and $a(t)=\frac{\gamma K}{t}$. Hence case (iii) of Theorem 1 or 2 applies, with $m=-1$ and $e^{A(t)}=t^{\gamma K}$. In the proofs, the minimum value of the integral $\int_{t_{1}}^{t_{2}} e^{-m A}|\dot{x}|^{q} d t$ was sought, subject 
to the constraints $t_{2}>t_{1}>0$ and $x\left(t_{1}\right)=x_{1}, x\left(t_{2}\right)=x_{2}$. For the case at hand, this minimum occurs at the path

(4.6a) $\quad x(t)=\left(\frac{x_{2}-x_{1}}{t_{2}^{\delta}-t_{1}^{\delta}}\right) t^{\delta}+\left(\frac{x_{1} t_{2}^{\delta}-x_{2} t_{1}^{\delta}}{t_{2}^{\delta}-t_{1}^{\delta}}\right), \quad$ if $0 \neq \delta:=(2-p) K+1$

and at

$$
\begin{aligned}
x(t)= & \left(\frac{x_{2}-x_{1}}{\log t_{2}-\log t_{1}}\right) \log t \\
& +\left(\frac{x_{1} \log t_{2}-x_{2} \log t_{1}}{\log t_{2}-\log t_{1}}\right), \quad \text { if }(2-p) K+1=0 .
\end{aligned}
$$

The minimum value of the integral in question is then $I^{1-q}$, where

$$
I=\frac{t_{2}^{\delta}-t_{1}^{\delta}}{\delta} \text { if } 0 \neq \delta:=(2-p) K+1
$$

and

$$
I=\log t_{2}-\log t_{1} \quad \text { if }(2-p) K+1=0 .
$$

These results may be collected as follows:

Theorem 3. Let $p>2\left(\frac{n}{n+1}\right)$ and $f$ be a nonnegative solution of (4.3) which is locally Lipshitz on an open convex set $\Omega \times\left(t_{1}, t_{2}\right)$ of $\mathbb{R}^{n} \times(0, \infty)$. Then

$$
f\left(x_{2}, t_{2}\right) \geq\left(\frac{t_{1}}{t_{2}}\right)^{\gamma K}\left[f\left(x_{1}, t_{1}\right)-\xi\left|x_{2}-x_{1}\right|^{q} I^{1-q} t_{1}^{-\gamma K}\right],
$$

for any $x_{1}, x_{2}$ in $\Omega$ and $t_{2}>t_{1}>0$. Here, $\gamma:=\frac{p-2}{p-1}, q:=\frac{p}{p-1}, \xi:=$ $\frac{1}{q}\left(\frac{1}{p}\right)^{q-1}$, and $I$ is given by (4.7a) or (4.7b), depending on whether the quantity $\delta:=(2-p) K+1$ is zero or nonzero.

Whenever $p>2,(4.8)$ and (4.2) combine to give a lower bound on the solution $u$ of the original $p$-diffusion equation:

$$
u^{\gamma}\left(x_{2}, t_{2}\right) \geq\left(\frac{t_{1}}{t_{2}}\right)^{\gamma K}\left[u^{\gamma}\left(x_{1}, t_{1}\right)-\gamma \xi\left|x_{2}-x_{1}\right|^{q} I^{1-q} t_{1}^{-\gamma K}\right]
$$

for any $x_{1}, x_{2}$ in $\Omega$ and $t_{2}>t_{1}>0$.

\section{Pointwise UPPER bOUNDS AND HAMILTON-JACOBI EQUATIONS}

The results of $\S 3$ provided pointwise lower bounds for solutions of differential inequalities of the form (3.1). Positive solutions $f$ of the transformed porous medium equation (2.3) with $M<1$ do not obey (3.1) but instead obey an inequality of the form

$$
\frac{\partial f}{\partial t}+a(t) f \leq \frac{-C|\nabla f|^{p}}{f^{r}}
$$

where $a$ is locally integrable and $C$ is a positive constant. The following result shows that pointwise upper bounds can be obtained for positive (if $r \neq 0$ ) or nonnegative (if $r=0$ ) solutions of (5.1). 
Theorem 4. Let $\Omega$ be an open convex set in $\mathbb{R}^{n}$; let $f: \Omega \times(0, T) \rightarrow[0, \infty)$ be a function which obeys the conditions (F1)-(F4) listed after (3.15), and is a distributional solution of (5.1) with $p>1$. For all $x_{1}, x_{2}$ in $\Omega$ and $0<t_{1}<$ $t_{2}<T$, we have the following inequalities:

(i) when $r=p-1$, then

$$
f\left(x_{2}, t_{2}\right) \leq \frac{e^{A\left(t_{1}\right)}}{e^{A\left(t_{2}\right)}} f\left(x_{1}, t_{1}\right) e^{\xi\left|x_{2}-x_{1}\right|^{q} /\left(t_{2}-t_{1}\right)^{q-1}}
$$

holds for positive $f$;

(ii) when $r>p-1$, then

$$
\left[f\left(x_{2}, t_{2}\right)\right]^{-m} \geq \frac{e^{m A\left(t_{2}\right)}}{e^{m A\left(t_{1}\right)}}\left(\left[f\left(x_{1}, t_{1}\right)\right]^{-m}-m \xi\left|x_{2}-x_{1}\right|^{q} I^{1-q} e^{m A\left(t_{1}\right)}\right)
$$

holds for positive $f$ and, whenever the quantity inside the large parentheses is nonnegative, we have

$$
f\left(x_{2}, t_{2}\right) \leq \frac{e^{A\left(t_{1}\right)}}{e^{A\left(t_{2}\right)}} f\left(x_{1}, t_{1}\right)\left(1-m \xi\left|x_{2}-x_{1}\right|^{q} I^{1-q}\left[f\left(x_{1}, t_{1}\right)\right]^{m} e^{m A\left(t_{1}\right)}\right)^{\frac{-1}{m}}
$$

(iii) when $r<p-1$, then

$$
\left[f\left(x_{2}, t_{2}\right)\right]^{|m|} \leq\left(\frac{e^{A\left(t_{1}\right)}}{e^{A\left(t_{2}\right)}}\right)^{|m|}\left(\left[f\left(x_{1}, t_{1}\right)\right]^{|m|}+\frac{|m| \xi\left|x_{2}-x_{1}\right|^{q} I^{1-q}}{e^{|m| A\left(t_{1}\right)}}\right)
$$

holds for nonnegative $f$ and, in case $f$ is positive, we have

$$
f\left(x_{2}, t_{2}\right) \leq \frac{e^{A\left(t_{1}\right)}}{e^{A\left(t_{2}\right)}} f\left(x_{1}, t_{1}\right)\left(1+\frac{|m| \xi\left|x_{2}-x_{1}\right|^{q} I^{1-q}}{\left[f\left(x_{1}, t_{1}\right)\right]^{|m|} e^{|m| A\left(t_{1}\right)}}\right)^{\frac{1}{|m|}} .
$$

The quantities $q, m, \xi, A$, and $I$ are as in Theorem 1 . Namely, $q:=$ $\frac{p}{p-1}$ is the conjugate index to $p, m:=\frac{r}{p-1}-1, \xi:=\frac{1}{q}\left(\frac{1}{p C}\right)^{q-1}, A(t)$ is any antiderivative of $a(t)$, and $I:=\int_{t_{1}}^{t_{2}} e^{m(p-1) A(t)} d t$.

Proof. The proof of this parallels that of Theorem 1. Let $W$ be any continuous vector field defined on $\Omega \times(0, T)$, and add $(\nabla f) \cdot W-\frac{1}{q}|\phi W|^{q}$, with $\phi:=$ $\left(\frac{f^{\prime}}{p C}\right)^{\frac{1}{p}}$, to both sides of (5.1). The right-hand side of the resulting inequality simplifies to the quantity $-\left(\frac{p C}{f^{r}}\right)\left(\frac{1}{p}|Y|^{p}-Y \cdot Z+\frac{1}{q}|Z|^{q}\right)$ with $Y=\nabla f$ and $Z=\left(\frac{f^{r}}{p C}\right) W$. By (3.5), this is pointwise nonpositive on $\Omega \times(0, T)$; so we have

$$
\frac{\partial f}{\partial t}+a(t) f+(\nabla f) \cdot W-\xi f^{r /(p-1)}|W|^{q} \leq 0, \quad \xi:=\frac{1}{q}\left(\frac{1}{p C}\right)^{q-1},
$$

for any vector field $W$ defined on $\Omega \times(0, T)$.

Given $x_{1}, x_{2}$ in $\Omega$, let $\left\{x(t): t_{1} \leq t \leq t_{2}\right\}$ be a $C^{1}$ curve in $\Omega$ with $x\left(t_{1}\right)=x_{1}$ and $x\left(t_{2}\right)=x_{2}$. Choose $W$ in $(5.5)$ to be $\dot{x} ;$ then the fact that the chain rule (3.15) holds implies that

$$
\begin{aligned}
& \frac{d}{d t} f(x(t), t)+a(t) f(x(t), t) \\
& \quad \leq \xi[f(x(t), t)]^{m+1}|\dot{x}(t)|^{q}, \quad m+1:=\frac{r}{p-1} .
\end{aligned}
$$

There are three cases $(m=0, m>0, m<0)$ to which we specialize (5.6). The resulting inequalities are respectively obtainable from (3.8), (3.10), and 
(3.13) by reversing the inequalitiy signs there and replacing $\xi$ with $-\xi$. The subsequent optimizations and manipulations are as in the proof of Theorem 1.

It is worth noting that (5.1) also appears directly as a Hamilton-Jacobi equation of the form

$$
\frac{\partial f}{\partial t}+H(t, f, \nabla f)=0,
$$

with

$$
H(t, f, \nabla f)=a(t) f+\frac{C|\nabla f|^{p}}{f^{r}}, \quad C>0 .
$$

A common convex Hamiltonian has $r=0, p>1$, and part (iii) of Theorem 4 provides pointwise upper bounds on nonnegative solutions of (5.7), (5.8). When $r=0$ but $p=1$, part (i) of that theorem provides some qualitatively different bounds.

\section{THE POROUS MEDIUM EQUATION REVISITED}

The results described in the last section can be applied to the porous medium equation (2.1) in the case where $1>M>M_{0}(n):=\max \left\{0,1-\frac{2}{n}\right\}$ to produce upper bounds on

$$
g:=\left(\frac{M}{1-M}\right) u^{M-1} .
$$

Since $M<1$, these give upper bounds on $u^{M-1}$, which in turn yield lower bounds on $u$. This, together with $\S 2$, show that for all values of $M>M_{0}(n)$ there are lower bounds on the positive/nonnegative solutions of (2.1).

Theorem 5. Suppose $M>M_{0}(n)$ and $u$ is a nonnegative (when $M>1$ ) or positive (when $M \leq 1$ ) weak solution of $\frac{\partial u}{\partial t}=\Delta\left(u^{M}\right)$ which obeys the conditions (F1)-(F4) listed after (3.15). Define $f$ by (2.2) when $M>1$ and $g$ by (6.1) when $M_{0}(n)<M<1$. For all $x_{1}, x_{2}$ in $\Omega$ and $0<t_{1}<t_{2}<\infty$, we have the following inequalities:

(A) when $M>1$, then

$$
f\left(x_{2}, t_{2}\right) \geq\left(\frac{t_{1}}{t_{2}}\right)^{\mu}\left[f\left(x_{1}, t_{1}\right)-\frac{1}{4} \frac{\delta\left|x_{2}-x_{1}\right|^{2}}{t_{2}^{\delta}-t_{1}^{\delta}} \frac{1}{t_{1}^{\mu}}\right]
$$

and, equivalently,

$$
u^{M-1}\left(x_{2}, t_{2}\right) \geq\left(\frac{t_{1}}{t_{2}}\right)^{\mu}\left[u^{M-1}\left(x_{1}, t_{1}\right)-\left(\frac{M-1}{M}\right) \frac{1}{4} \frac{\delta\left|x_{2}-x_{1}\right|^{2}}{t_{2}^{\delta}-t_{1}^{\delta}} \frac{1}{t_{1}^{\mu}}\right]
$$

(B) when $M=1$, then

$$
u\left(x_{2}, t_{2}\right) \geq\left(\frac{t_{1}}{t_{2}}\right)^{\frac{n}{2}} u\left(x_{1}, t_{1}\right) e^{-\left[\left|x_{2}-x_{1}\right|^{2} /\left(t_{2}-t_{1}\right)\right] / 4} ;
$$

(C) when $M_{0}(n)<M<1$, then

$$
g\left(x_{2}, t_{2}\right) \leq\left(\frac{t_{2}}{t_{1}}\right)^{\nu}\left[g\left(x_{1}, t_{1}\right)+\frac{1}{4} \frac{\delta\left|x_{2}-x_{1}\right|^{2}}{t_{2}^{\delta}-t_{1}^{\delta}} t_{1}^{\nu}\right]
$$


and, equivalently,

$$
u^{1-M}\left(x_{2}, t_{2}\right) \geq\left(\frac{t_{1}}{t_{2}}\right)^{\nu}\left[u^{M-1}\left(x_{1}, t_{1}\right)+\left(\frac{1-M}{M}\right) \frac{1}{4} \frac{\delta\left|x_{2}-x_{1}\right|^{2}}{t_{2}^{\delta}-t_{1}^{\delta}} t_{1}^{\nu}\right]^{-1}
$$

Here, $k:=\frac{1}{\left(M-1+\frac{2}{n}\right)}, \quad \mu:=(M-1) k, \quad \nu:=(1-M) k, \quad \delta:=1-\mu=$ $1+\nu=\frac{2 k}{n}$.

Proof. Cases (A) and (B) have already been treated in $\S 2$. As for case (C), it follows from $(6.1),(2.2)-(2.4)$ that

$$
\frac{\partial g}{\partial t}=(1-M) g \Delta g-|\nabla g|^{2}
$$

and

$$
\Delta g \leq \frac{k}{t} .
$$

Multiplying (6.6) by $(1-M) g$ and using (6.5), it follows that

$$
\frac{\partial g}{\partial t}-[(1-M) k] \frac{g}{t} \leq-|\nabla g|^{2} .
$$

This is an inequality of the type (5.1) with $r=0, p=2, C=1$, and $a(t)=$ $-\left[\frac{(1-M) k}{t}\right]$. Thus we are in case (iii) of Theorem 4 , with $q=2, m=-1$, $\xi=\frac{1}{4}, A(t)=\log \left(t^{-\nu}\right)$, and $I=\left(t_{2}^{\delta}-t_{1}^{\delta}\right) / \delta$. Equation (5.4a) then becomes $(6.4 a)$.

\section{ACKNOWLEDGMENTS}

We thank D. Aronson and B. Keyfitz for supplying us with references [1] and [5]; W. Fitzgibbon for clarifying a point in [2]; and R. Hardt and K. Uhlenbeck for suggesting that we compare our results with [4].

\section{REFERENCES}

1. D. G. Aronson, The porous medium equation, Nonlinear Diffusion Problems (A. Fasano and M. Primicerio, eds.), Lecture Notes in Math., vol. 1224, Springer-Verlag, Berlin, 1986.

2. D. G. Aronson and P. Bénilan, Régularité des solutions de l'équation des milieux poreux dans $\mathbb{R}^{n}$, C. R. Acad. Sci. Paris Sér. I Math. 288 (1979), 103-105.

3. E. DiBenedetto, Topics in quasilinear degenerate and singular parabolic equations, Vorlesungsreihe no. 20, SFB 256, Universität Bonn, 1991.

4. E. DiBenedetto and A. Friedman, Hölder estimates for nonlinear degenerate parabolic systems, J. Reine Angew. Math. 357 (1985), 1-22.

5. J. R. Esteban and J. L. Vásquez, Regularity of nonnegative solutions of the p-Laplacian parabolic equation, $1^{a}$ Reunion Hispano-Italiana de Analisis No Lineal y Matematica Aplicada, 1989, pp. 87-92.

6. D. Gilbarg and N. S. Trudinger, Elliptic partial differential equations of second order, 2nd ed., Springer, New York, 1983.

7. R. Hamilton, Lecture given at the NSF Summer Institute on Differential Geometry, UCLA, 1990.

8. A. Harnack, Grundlagen der Theorie des Logarithmischen Potentials, Teubner, Leipzig, 1887.

9. P. Li and S. T. Yau, On the parabolic kernel of the Schrödinger operator, Acta Math. 156 (1986), 153-201. 
10. J. Moser, A Harnack inequality for parabolic differential equations, Comm. Pure Appl. Math. 17 (1964), 101-134.

11. M. Marcus and V. J. Mizel, Absolute continuity on tracks and mappings of Sobolev spaces, Arch. Rational Mech. Anal. 45 (1972), 294-320.

Department of Mathematics, University of Houston, Houston, TeXas 77204-3476

E-mail address: auch@uh.edu

E-mail address: bao@uh.edu 